\title{
Mean End-to-End Distance of a Polymer Confined between Two Interacting Plates
}

\author{
Kohzoh SHIOKAwA \\ Department of Applied Science, Faculty of Engineering, \\ Kyushu University, Hakozaki, Higashi-ku, \\ Fukuoka 812, Japan
}

(Received February 4, 1993)

\begin{abstract}
The mean end-to-end distance of a polymer confined between two plates, and interaction with the plates, were studied in the presence of excluded volume interaction. The polymer surface interaction is included exactly in the unperturbed distribution function of the confined polymer as the boundary condition. The excluded volume interaction is introduced perturbatively using the homotopy parameter expansion method. The component of the mean square end to end distance parallel to the plates is calculated as the function of $D W$ and $D /\left\langle R_{z}^{2}\right\rangle_{\infty}^{1 / 2}$, where $D$ is the interplate distance, $W$ is the the polymer-plate interaction parameter and $\left\langle R_{z}^{2}\right\rangle_{\infty}$ is the component of the mean square end to end distance perpendicular to the plates in an unconfined state. It was found that the attractive interaction between the polymer and the plates has a larger effect on chain dimensions than the repellent interaction between them. The parallel components of the mean end-to-end distance of the chain where one end is fixed at an assigned position is also calculated.
\end{abstract}

KEY WORDS End-to-End Distance / Confined Polymer / Slit / Slab / Excluded Volume / Renormalization Group Theory / Homotopy Parameter Expansion / Adsorption /

The conformational properties of a polymer interacting with a surface are relevant to many practical problems such as adhesion, stabilization of colloid particles and reinforcement. Many theoretical studies have been carried out. ${ }^{1}$ A polymer confined between two plates, and interacting with the plates, has also been studied. However, most of these reports deal with systems in which one end, or else some parts of the polymers are anchored on the plate. The effects of the polymer surface interaction on the conformational properties were studied by DiMarzio and Rubin ${ }^{2}$ using lattice models and by Chan, Davis and Richmond ${ }^{3}$ using a continuum model. However, the full dependencies of the polymer surface interaction from an unconfined state to a confined state have not yet been studied.

The excluded volume interaction has a strong influence on chain dimensions not only in an unconfined state but also in a confined state.The excluded volume interaction further complicates the calculation of chain dimensions. There are a few studies dealing with the effects of both the excluded volume interaction and polymer surface interaction on chain conformations. Daoud and de Gennes ${ }^{4}$ and Turban ${ }^{5}$ discussed the dimensions of a confined chain using scaling arguments. However, they did not study the effect of polymer plate interaction. Wang, Nemirovsky and Freed ${ }^{6}$ investigated chain dimensions using the $\varepsilon$ expansion method for cases of a polymer confined between two perfectly repellent plates and between two reflecting plates. The present author derived the full dependence of the chain dimensions of a confined chain on the interplate distance, using the homotopy parameter ex- 
pansion method ${ }^{7}$ and mean field theory ${ }^{8}$ for cases in which a polymer is confined between two perfectly repellent plates.

In this paper, we studied the full dependence of the mean end-to-end distance of a confined polymer on the interplate distance, and on the polymer surface interaction in the presence of the excluded volume. The polymer surface interaction is included exactly within the distribution functions of the unperturbed polymer confined between two plates. The excluded volume interaction is then introduced perturbatively using the homotopy parameter expansion method proposed by Oono. ${ }^{9}$

\section{MODEL}

A model chain consists of $N_{0}$ freely jointed bonds of a unit length. The interplate distance, $D$, is much larger than unity, so the continuous polymer chain model can be utilized. The $z$ axis is taken perpendicular to the plates and the $x$ and $y$ axes are taken parallel to the plates. The polymer chain is confined between $z=-D / 2$ and $D / 2$.

The probability distribution function of an unperturbed chain which starts at $\boldsymbol{R}^{\prime}$ and ends at $\boldsymbol{R}$, satisfies the following differential equation.

$$
\left(\frac{\partial}{\partial N_{0}}-\frac{1}{6} \nabla^{2}\right) G_{0}\left(\boldsymbol{R}, \boldsymbol{R}^{\prime} ; N_{0}\right)=\delta\left(\boldsymbol{R}-\boldsymbol{R}^{\prime}\right) \delta\left(N_{0}\right)
$$

On the atomic scale, interaction between the polymer and the plates depends on the local conformations of the polymer at the plates, and on the fine structures of the plate surfaces. However it is too difficult to introduce their contributions in the distribution function. We disregard these fine structures, and assume that the interaction has effects only on bonds in the vicinity of the plate surfaces. Thus the interaction is introduced as a boundary condition for eq 1 according to deGennes ${ }^{10}$

$$
\left(\partial G_{0} / \partial z\right) / G_{0}=\mp W \quad \text { at } \quad z= \pm D / 2
$$

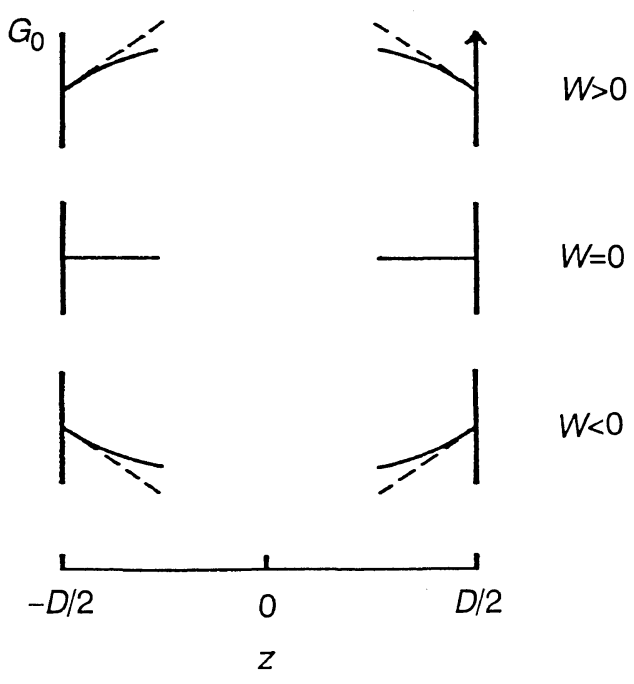

Figure 1. Schematic diagram of the polymer plate interaction parameter. Top, repellent surface; middle, reflecting surface; bottom, attractive surface.

where $W$ is the polymer plate interaction parameter per unit length. The schematic diagram of eq 2 is given in Figure 1. For positive values of $W$, segment concentration in the bulk state is richer than that on the surface. A positive value of $W$ thus means that the polymer surface interaction is repellent. In contrast, a negative value of $W$ corresponds to attractive interaction.

From eq 1 and $2, G_{0}$ is translationally invariant for $x$ and $y$ directions. Then $G_{0}$ can be decomposed as

$$
\begin{aligned}
& G_{0}\left(\boldsymbol{R}, \boldsymbol{R}^{\prime} ; N_{0}\right) \\
& \quad=G_{0 x}\left(R_{x} ; N_{0}\right) G_{0 y}\left(R_{y} ; N_{0}\right) G_{0 z}\left(z, z^{\prime} ; N_{0}\right)
\end{aligned}
$$

where $R_{x}$ and $R_{y}$ are the components of the end-to-end vector parallel to the plates. For an unperturbed chain, $G_{0 x}$ and $G_{0 y}$ are Gaussian functions.

Solving eq 1 under the boundary conditions of eq 2, we get the component of distribution function perpendicular to the plates, $G_{0 z}$ as follows: 


$$
\begin{aligned}
& G_{0 z}\left(z, z^{\prime} ; N_{0}\right) \\
& =\frac{2}{D}\left\{\sum_{k} A_{k} \cos \left(\frac{a_{k} z}{D}\right) \cos \left(\frac{a_{k} z^{\prime}}{D}\right) \exp \left(-a_{k}^{2} d_{0}\right)\right. \\
& +\sum_{k} B_{k} \sin \left(\frac{b_{k} z}{D}\right) \sin \left(\frac{b_{k} z^{\prime}}{D}\right) \exp \left(-b_{k}^{2} d_{0}\right) \\
& +A \cosh \left(\frac{a z}{D}\right) \cosh \left(\frac{a z^{\prime}}{D}\right) \exp \left(a^{2} d_{0}\right) \\
& \left.+B \sinh \left(\frac{b z}{D}\right) \sinh \left(\frac{b z^{\prime}}{D}\right) \exp \left(b^{2} d_{0}\right)\right\}
\end{aligned}
$$

where $d_{0}=N_{0} / 6 D^{2}$. The coefficients $a_{k}, b_{k}, a$, and $b$ are determined by the following transcendental equations, where $A_{k}, B_{k}, A$, and $B$ are normalization constants:

$\tan \left(a_{k} / 2\right)=D W / a_{k} ; \quad A_{k}=1 /\left(1+\sin \left(a_{k}\right) / a_{k}\right)(5 \mathrm{a})$

$\cot \left(b_{k} / 2\right)=-D W / b_{k} ; B_{k}=1 /\left(1-\sin \left(b_{k}\right) / b_{k}\right)(5 b)$

$\tanh (a / 2)=-D W / a ; A=1 /(\sinh (a) / a+1) \quad(5 \mathrm{c})$

$\operatorname{coth}(b / 2)=-D W / b ; B=1 /(\sinh (b) / b-1) \quad(5 \mathrm{~d})$

The nature of the roots, $a_{k}$ and $b_{k}$ is demonstrated in Figure 2. This illustrates the

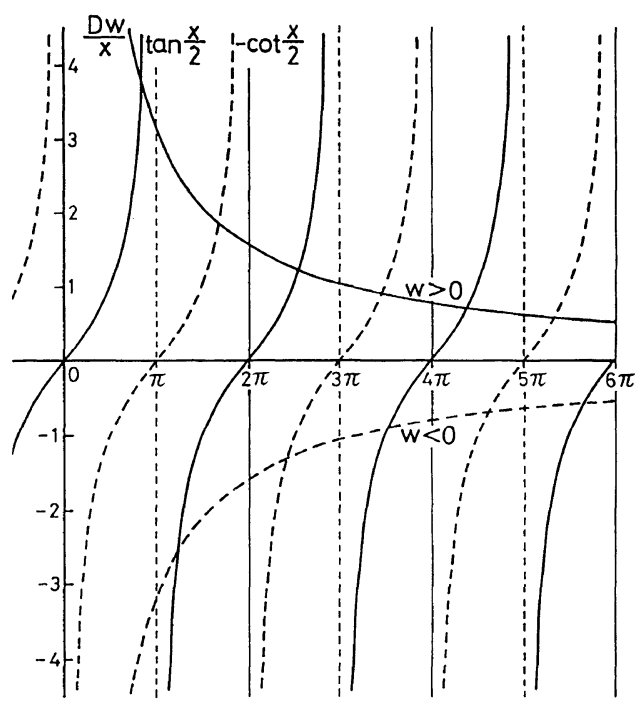

Figure 2. Graphical solutions of transcendental equations. Solid curves, $\tan (x / 2)$; Dotted curves; $-\cot (x / 2)$. Intersecting points correspond to solutions of eq $5 \mathrm{a}$ and $5 b$. curves corresponding to the right- and left-hand sides of eq $5 \mathrm{a}$ and $5 \mathrm{~b}$, respectively. For given $D W$, the values of abscissas of intersecting points correspond to $a_{k}$ or $b_{k}$ for $k=0,1,2, \cdots$. The values of $a_{k}$ and $b_{k}$ are plotted against $D W$ in Figures $3 \mathrm{a}$ and $3 \mathrm{~b}$, according to the sign of $D W$. They increase with $D W$. There is no real root of $a_{0}$ when $D W<0$, or $b_{1}$ when $D W<-2$. The nature of eq $5 \mathrm{c}$ and $5 \mathrm{~d}$ is illustrated in Figure 4. As for the roots of eq $5 \mathrm{c}, a$ is real when $D W$ is negative, and $b$ is real when $D W$ is less than -2 . The

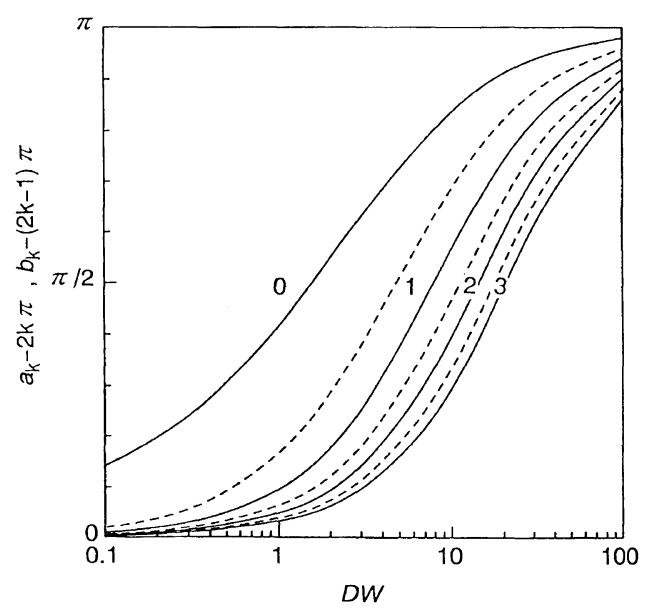

(a)

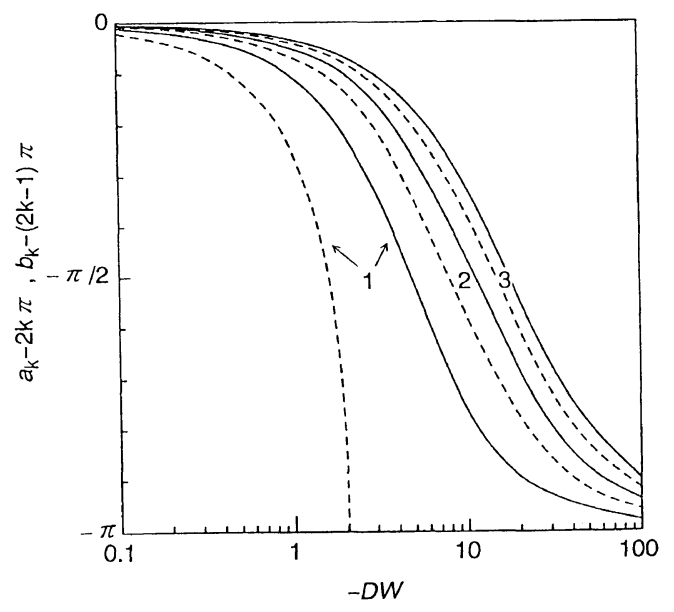

(b)

Figure 3. $a_{k}$ and $b_{k}$ as functions of $D W$. Numerical values in the figure are the values of $k$. 


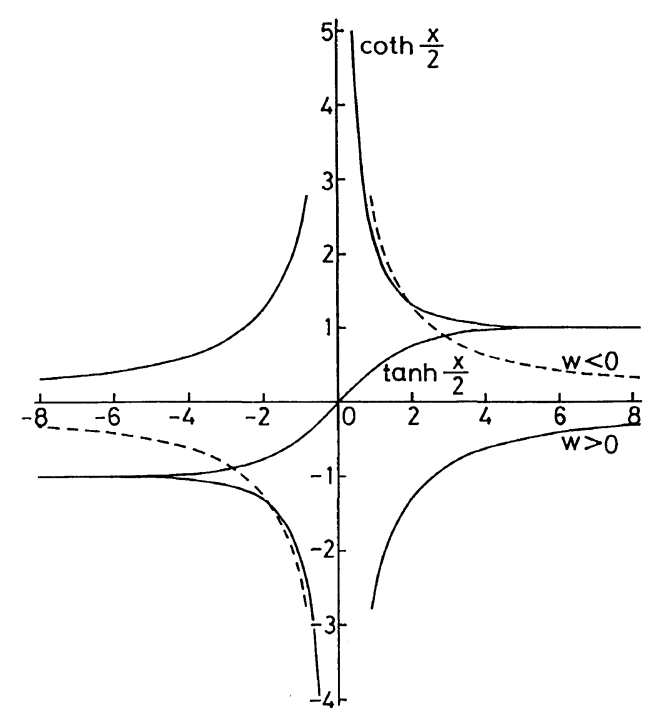

Figure 4. Graphical solutions of transcendental equation. $\tanh (x / 2)$ and $\operatorname{coth}(x / 2)$ are plotted against $x$. Intersecting points of these curves and the curve which represents $D W / x$ give solutions to eq $5 \mathrm{c}$ and $5 \mathrm{~d}$.

values of $a$ and $b$ are shown as functions of $-D W$ in Figure 5. Both values of $a$ and $b$ approach $-D W$ as $-D W$ increases. If we set $a_{0}=i a$ and $b_{1}=i b$, the last two terms in eq 4 can be replaced by the first terms of the first and second sums in eq 4, respectively. Hereafter, we use these notations for convenience.

It is of interest that the parameters, $a_{k}, b_{k}$ depend on the value of $D W$, but are independent of chain dimensions. However the distribution function of the confined chain is determined not only on $D W$ but also $d_{0}$, the square of the ratio of the radius of gyration of the unconfined chain to the interplate distance.

The Hamiltonian expression proposed by Oono was depicted as ${ }^{8}$

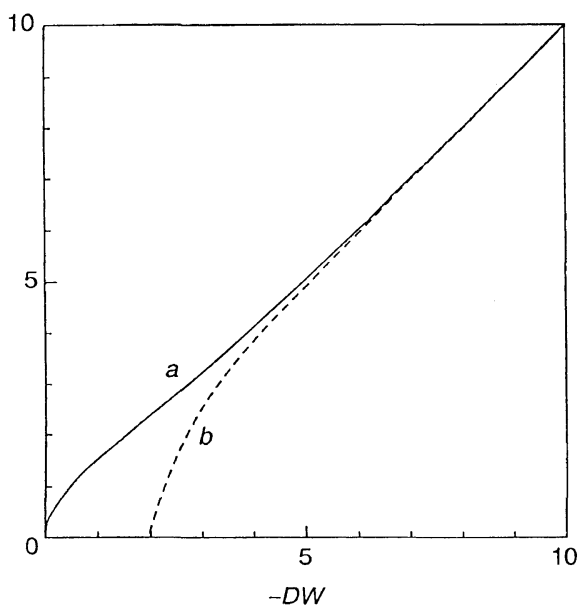

Figure 5. $a$ and $b$ as functions of $-D W$.

$$
\begin{aligned}
H= & \frac{1}{2} \int_{0}^{N_{0}} \mathrm{~d} S\left(\frac{\mathrm{d}}{\mathrm{d} S} C(S)\right)^{2} \\
& +\frac{1}{2} v_{0} \int_{0}^{N_{0}} \mathrm{~d} S \int_{0}^{N_{0}} \mathrm{~d} S^{\prime} \delta\left(C(S)-C\left(S^{\prime}\right)\right) \\
& \left|S-S^{\prime}\right|>c \quad \times l\left(S, S^{\prime}\right)^{\theta-1 / 2}
\end{aligned}
$$

where $C(S)$ designates the spatial position of the polymer segments at a contour length $S$ along the chain, $v_{0}$ is the excluded volume parameter, $l\left(S, S^{\prime}\right)$ is the shortest contour length between $C(S)$ and $C\left(S^{\prime}\right)$ along the chain, assuming both ends are connected with each other, $\theta$ is the homotopy parameter, and $c$ is the cut-off distance which is introduced to eliminate the self interaction of segments.

Using eq 6 , the first order perturbation expansion of the distribution function of the perturbed chain, which starts at $\boldsymbol{R}^{\prime}$ and ends at $\boldsymbol{R}$, with respect to $v_{0}$ is given as

$$
\begin{aligned}
G_{b}\left(\boldsymbol{R}, \boldsymbol{R}^{\prime} ; N_{0}\right)= & G_{0}\left(\boldsymbol{R}, \boldsymbol{R}^{\prime} ; N_{0}\right)-v_{0} \int \mathrm{d} S\left[\min \left(S, N_{0}-S\right)\right]^{\theta-1 / 2} \\
& \times \int \mathrm{d} S^{\prime} \int \mathrm{d} \boldsymbol{R}^{\prime \prime} G_{0}\left(\boldsymbol{R}^{\prime \prime}, \boldsymbol{R}^{\prime} ; S^{\prime}\right) G_{0}\left(\boldsymbol{R}^{\prime \prime}, \boldsymbol{R}^{\prime \prime} ; S\right) G_{0}\left(\boldsymbol{R}, \boldsymbol{R}^{\prime \prime} ; N_{0}-S-S^{\prime}\right) \\
= & G_{0 x}\left(R_{x} ; N_{0}\right) G_{0 y}\left(R_{y} ; N_{0}\right) G_{0 z}\left(z, z^{\prime} ; N_{0}\right)
\end{aligned}
$$




$$
\begin{aligned}
& -v_{0} \int \mathrm{d} S(3 / 2 \pi S) G_{0 x}\left(R_{x} ; N_{0}-S\right) G_{0 y}\left(R_{y} ; N_{0}-S\right) \\
& \times I\left(z, z^{\prime} ; S, N_{0}\right)\left[\min \left(S, N_{0}-S\right)\right]^{\theta-1 / 2}
\end{aligned}
$$

where $S$ is the contour length of the loop in eq $6 . I$ is given as

$$
\begin{aligned}
I\left(z, z^{\prime} ; S, N_{0}\right)= & \int_{0}^{N_{0}-S} \mathrm{~d} S^{\prime} \int_{-D / 2}^{D / 2} \mathrm{~d} z^{\prime \prime} G_{0 z}\left(z^{\prime \prime}, z^{\prime} ; S^{\prime}\right) G_{0 z}\left(z^{\prime \prime}, z^{\prime \prime} ; S\right) G_{0 z}\left(z, z^{\prime \prime} ; N_{0}-S-S^{\prime}\right) \\
= & 12 d_{0}(1-t) \\
& \times\left\{\sum_{k} \sum_{l} A_{k} A_{l} \cos \left(a_{k} z / D\right) \cos \left(a_{l} z^{\prime} / D\right) K_{0}\left(a_{k}^{2} d_{0}(1-t), a_{l}^{2} d_{0}(1-t)\right)\right. \\
& \times\left[\sum_{m} A_{m} J_{k l m}^{c c} \exp \left(-a_{m}^{2} d_{0} t\right)+B_{m} J_{k l m}^{c s} \exp \left(-b_{m}^{2} d_{0} t\right)\right] \\
+ & \sum_{k} \sum_{l} B_{k} B_{l} \sin \left(b_{k} z / D\right) \sin \left(b_{l} z^{\prime} / D\right) K_{0}\left(b_{k}^{2} d_{0}(1-t), b_{l}^{2} d_{0}(1-t)\right) \\
& \left.\times\left[\sum_{m} A_{m} J_{k l m}^{s c} \exp \left(-a_{m}^{2} d_{0} t\right)+B_{m} J_{k l m}^{s s} \exp \left(-b_{m}^{2} d_{0} t\right)\right]\right\}
\end{aligned}
$$

where

$$
K_{0}(a, b)=(\exp (-b)-\exp (-a)) /(a-b)
$$

and $t=S / N_{0}$. The explicit forms of $J_{k l m}$ are given in the Appendix.

The value of $G_{b}$ depends on the value of the cut off distance, $c$. In the limit to approach $c$ to 0 , the value of $G_{0}$ diverges as the value of $\theta$ approaches to 0 . Removal of the unphysical sensitive dependence on the cut off distance is done by renormalization group analysis, as shown in the next section.

\section{MEAN SQUARE END-to-END DISTANCE}

\section{Bare Perturbation}

The component of the mean square end-toend distance parallel to the plates, $\left\langle R_{x}^{2}\right\rangle$ is given as

$$
\left\langle R_{x}^{2}\right\rangle=\frac{N_{0}}{3}+\frac{1}{3} \frac{3}{2 \pi} v_{0} \frac{\int \mathrm{d} z \int \mathrm{d} z^{\prime} I_{x}\left(z, z^{\prime}\right)}{\int \mathrm{d} z \int \mathrm{d} z^{\prime} G_{0 z}\left(z, z^{\prime} ; N_{0}\right)}
$$

where

$$
\begin{aligned}
& I_{x}\left(z, z^{\prime}\right)= \\
& \int \mathrm{d} S I\left(z, z^{\prime} ; S, N_{0}\right)\left[\min \left(S, N_{0}-S\right)\right]^{\theta-1 / 2}
\end{aligned}
$$

Introducing eq 8 into eq 11 , integrations are performed, so that

$$
\begin{aligned}
& \int \mathrm{d} z \int \mathrm{d} z^{\prime} I_{x}\left(z, z^{\prime}\right)=D^{2} N_{0}^{\theta-1 / 2} 12 d_{0} \\
& \times\left\{\sum_{k} A_{k} f^{2}\left(a_{k}\right) g_{x}\left(a_{k}^{2} d_{0}\right)+\sum_{k} A_{k}^{2} f^{2}\left(a_{k}\right) T_{x k}^{c}\left(d_{0}\right)\right. \\
& \left.\quad+2 \sum_{k<l} \sum_{k} A_{k} A_{l} f\left(a_{k}\right) f\left(a_{l}\right) T_{x k l}^{c}\left(d_{0}\right)\right\}
\end{aligned}
$$




$$
\begin{aligned}
& g_{x}(x) \int_{0}^{1 / 2} \mathrm{~d} t(1-t) t^{\theta-1 / 2} \exp (-x(1-t)) H^{0}\left(d_{0} t\right) \\
&+\int_{1 / 2}^{1} \mathrm{~d} t(1-t)^{1 / 2} \exp (-x(1-t)) H^{0}\left(d_{0} t\right) \\
& H^{0}(x)= \sum_{m}\left[\exp \left(-a_{m}^{2} x\right)+\exp \left(-b_{m}^{2} x\right)\right] \\
& T_{x k}^{c}(\mathrm{~d})= \exp \left(-a_{k}^{2} d\right)\left\{\sum_{m} A_{m} J_{k m}^{c c} g_{x 1}\left(a_{m}^{2} d-a_{k}^{2} d\right)\right. \\
&\left.+\sum_{m} B_{m} J_{k m}^{c s} g_{x 1}\left(b_{m}^{2} d-a_{k}^{2} d\right)\right\} \\
& g_{x 1}(x)= \int_{0}^{1 / 2} \mathrm{~d} t(1-t) t^{-1 / 2} \exp (-x t) \\
&+\int_{1 / 2}^{1} \mathrm{~d} t(1-t)^{1 / 2} \exp (-x t) \\
&+\int_{1 / 2}^{1} \mathrm{~d} t(1-t)^{-1 / 2} \exp (-x t) \\
&+\sum_{m} B_{m} J_{k l m}^{c s}\left[\exp \left(-a_{l}^{2} d\right) g_{x 2}\left(b_{m}^{2} d-b_{l}^{2} d\right)\right. \\
& T_{x k l}^{c}(\mathrm{~d})=\left\{\sum _ { m } A _ { m } J _ { k l m } ^ { c c } \left[\exp \left(-a_{l}^{2} d\right) g_{x 2}\left(a_{m}^{2} d-a_{l}^{2} d\right)\right.\right. \\
&\left.-\exp \left(-a_{k}^{2} d\right) g_{x 2}\left(a_{m}^{2} d-a_{k}^{2} d\right)\right]
\end{aligned}
$$

The explicit forms of $J_{k m}$ are given in the Appendix. We put $\theta=0$ into eq 14, 17, and 19 , except for the first term in eq 14 , because these terms are regular at $\theta=0$.

$H^{0}(x)$ can be evaluated using the following approximation.

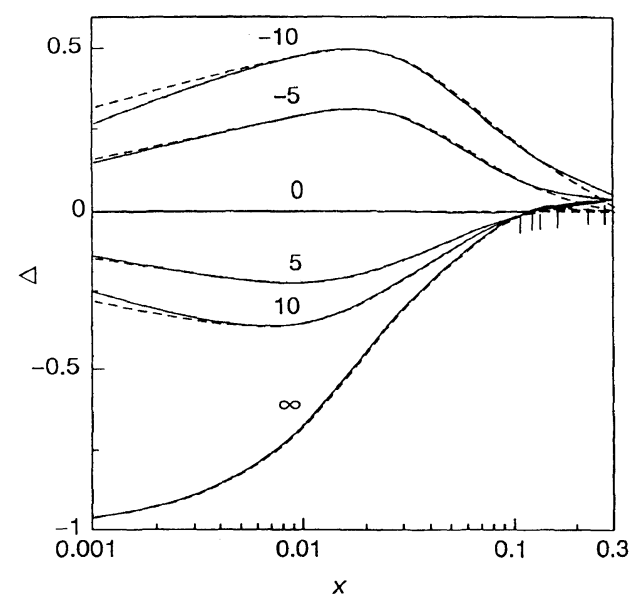

Figure 6. Verification of the approximation in eq 20 . Solid curves: $\Delta=\sum_{k}\left[\exp \left(-a_{k}^{2} x\right)+\exp \left(-b_{k}^{2} x\right)\right]-(\pi x)^{1 / 2}+$ $1 / 2+\exp \left(-\pi^{2} x\right)-\exp \left(-a_{0}^{2} x\right)-\exp \left(-b_{0}^{2} x\right)$. Dotted curves, $a x^{\mathrm{b}} \exp (-c x)$. The numerical values in the figure are the values of $D W$. Vertical bars represent the values of $x_{0}$. The order left to right corresponds to decreasing values of $D W$.

$H^{0}(x)= \begin{cases}(\pi x)^{-1 / 2} / 2+h_{0}(x)+h_{1}(x) & x<x_{0} \\ h_{1}(x) & x>x_{0}\end{cases}$

where

$$
\begin{aligned}
& h_{0}(x)=-\frac{1}{2}-\exp \left(-\pi^{2} x\right)+a x^{b} \exp (-c x) \\
& h_{1}(x)=\exp \left(-a_{0}^{2} x\right)+\exp \left(-b_{1}^{2} x\right)
\end{aligned}
$$

and where $a, b, c$, and $x_{0}$ are suitable chosen parameters.

In Figure $6, \Delta=H^{0}(x)-(\pi x)^{-1 / 2} / 2+1 / 2+$ $\exp \left(-\pi^{2} x\right)-h_{1}(x)$ is plotted against $x$ for various values of $D W$. The dotted curves indicate the corresponding values of $a x^{b}$ $\exp (-c x)$. Agreement of the solid and dotted curves is good for wide ranges of $x$. Equation 20 is suitable for an evaluation of the value of $H^{0}(x)$.

Substituting eq 20 into eq 14, and expanding the integrand with respect to $\theta$ around 0 , the integral up to the 0 -th order of $\theta$ can be evaluated as

$$
\begin{aligned}
g_{x}(x)= & \left(\pi d_{0}\right)^{-1 / 2} / 2 \exp (-x)\left[1 / \theta+g_{x 00}(x)\right] \\
& +g_{x 01}(x)
\end{aligned}
$$


where

$$
\begin{gathered}
g_{x 00}(x)=\int_{0}^{x p_{0}} \mathrm{~d} t[(\exp (t)-1) / t]+\ln \left(p_{0}\right) \\
\quad+\left[1-\exp \left(x p_{0}\right)\right] / x \\
\quad+\int_{1 / 2}^{p_{1}} \mathrm{~d} t(1-t)^{1 / 2} t^{-1 / 2} \exp (x t) \\
g_{x 01}(x)= \\
\int_{0}^{p_{0}} \mathrm{~d} t(1-t) t^{-1 / 2} h_{0}\left(d_{0} t\right) \exp (-x(1-t)) \\
+\int_{1 / 2}^{p_{1}} \mathrm{~d} t(1-t)^{1 / 2} h_{0}\left(d_{0} t\right) \exp (-x(1-t)) \\
+\int_{0}^{1 / 2} \mathrm{~d} t(1-t) t^{-1 / 2} h_{1}\left(d_{0} t\right) \exp (-x(1-t)) \\
+\int_{1 / 2}^{1} \mathrm{~d} t(1-t)^{1 / 2} h_{1}\left(d_{0} t\right) \exp (-x(1-t))(24)
\end{gathered}
$$

$p_{o}$ and $p_{1}$ are the parameters used to select the approximation for eq 20 , and are given as

$$
\begin{aligned}
& p_{0}=\min \left(x_{0} / d_{0}, 1 / 2\right) \\
& p_{1}=\max \left(1 / 2, \min \left(x_{0} / d_{0}, 1\right)\right)
\end{aligned}
$$

\section{Renormalization}

Equation 22 shows that $g_{x}$ is $\operatorname{singular}$ at $\theta=0$. This singularity must be adsorbed in the renormalization constants. We introduce a phenomenological number of bonds, $N$, and a renormalized excluded volume parameter, $u$. The renormalization constants are defined and expanded as

$$
\begin{array}{ll}
N=Z_{N} N_{0} & Z_{N}=1+B u \\
u=Z_{u} u_{0} & Z_{u}=1+o(u)
\end{array}
$$

where $u_{0}=(3 / 2 \pi)^{3 / 2} v_{0} L^{\theta}$ is the dimensionless excluded volume parameter, and $L$ is a phenomenological coarse-grain length. Substituting eq $26 \mathrm{a}$ and $26 \mathrm{~b}$ into eq 10 , and expanding up to the first order of $u$, the singularity in the limit of $\theta \rightarrow 0$ is adsorbed by setting $B=1 / \theta$.

This results in

$$
\begin{aligned}
\left\langle R_{x}^{2}\right\rangle= & \frac{N(N / L)^{u}}{3}\left\{1+u\left[F_{x 1}\left(d^{*}\right)\right.\right. \\
& \left.\left.+2\left(\pi d^{*}\right)^{1 / 2} F_{x 2}\left(d^{*}\right)\right]\right\}
\end{aligned}
$$

where

$$
\begin{aligned}
& F_{x 1}(d)= \\
& \left\{\sum_{k} A_{k} f^{2}\left(a_{k}\right) \exp \left(-a_{k}^{2} d\right) g_{x 00}\left(a_{k}^{2} d\right)\right\} \\
& /\left\{\sum_{k} A_{k} f^{2}\left(a_{k}\right) \exp \left(-a_{k}^{2} d\right)\right\} \\
& F_{x 2}(d)= \\
& \left\{\sum_{k} A_{k} f^{2}\left(a_{k}\right) g_{x 01}\left(a_{k}^{2} d\right)+\sum_{k} A_{k}^{2} f^{2}\left(a_{k}\right) T_{x k}^{c}(d)\right. \\
& \left.\quad+2 \sum_{k<l} \sum_{k} A_{l} f\left(a_{k}\right) f\left(a_{l}\right) T_{x k l}^{c}(d)\right\} \\
& /\left\{\sum_{k} A_{k} f^{2}\left(a_{k}\right) \exp \left(-a_{k}^{2} d\right)\right\}
\end{aligned}
$$

and

$$
d^{*}=N(N / L)^{u} / 6 D^{2}
$$

The stable fixed point $u^{*}$ is the same as that obtained in the previous paper, ${ }^{7}$ which is given as

$$
u^{*}=\theta / 4
$$

For a suitable long chain in a good solvent, we use $u^{*}$ instead of $u$.

At the limit of $D \rightarrow \infty(d \rightarrow 0)$, (as shown in the Discussions section), we get

$$
g_{x 00}(0)=g_{\infty}=-\ln 2-1+\pi / 4
$$

and for the parallel component of the mean square end-to-end distance in an unconfined state, we get

$$
\left\langle R_{x}^{2}\right\rangle_{\infty}=\frac{N(N / L)^{u}}{3}\left(1+(\theta / 4) g_{\infty}\right)
$$

The contribution of $(\pi d)^{1 / 2} F\left(d^{*}\right)$ is negligible. Finally, the following relation is obtained 


$$
\begin{aligned}
& \left\langle R_{x}^{2}\right\rangle \mid\left\langle R_{x}^{2}\right\rangle_{\infty}= \\
& 1+(\theta / 4)\left[F_{x 1}(d)-g_{\infty}+2(\pi d)^{1 / 2} F_{x 2}(d)\right]
\end{aligned}
$$

where

$$
d=\left\langle R_{z}^{2}\right\rangle_{\infty} / 2 D^{2}
$$

$\left\langle R_{z}^{2}\right\rangle$ is the perpendicular component of the mean square end-to-end distance, and the suffix $\infty$ indicates the value in an unconfined state.

\section{Mean Square End-to-End Distance of a Chain Where One End is Fixed}

If one end of a chain is fixed at $z$, the parallel component of the mean square end-to-end distance of the chain, $\left\langle R_{x}^{2}(z)\right\rangle$ is given as

$$
\left\langle R_{x}^{2}(z)\right\rangle=\frac{N_{0}}{3}+\frac{1}{3} \frac{3}{2 \pi} v_{0} \frac{\int \mathrm{d} z^{\prime} I_{x}\left(z, z^{\prime}\right)}{\int \mathrm{d} z^{\prime} G_{0 z}\left(z, z^{\prime} ; N_{0}\right)}
$$

In the same manner for $\left\langle R_{x}^{2}\right\rangle$, we get

$$
\begin{aligned}
\left\langle R_{x}^{2}(z)\right\rangle /\left\langle R_{x}^{2}\right\rangle_{\infty}= & 1+(\theta / 4)\left[F_{x 1}(z / D, d)-g_{\infty}\right. \\
& \left.+2(\pi d)^{1 / 2} F_{x 2}(z / D, d)\right]
\end{aligned}
$$

where

$$
\begin{gathered}
F_{x 1}(x, d)= \\
\left\{\sum_{k} A_{k} \cos \left(a_{k} x\right) f\left(a_{k}\right) \exp \left(-a_{k}^{2} d\right) g_{x 00}\left(a_{k}^{2} d\right)\right\} \\
/\left\{\sum_{k} A_{k} \cos \left(a_{k} x\right) f\left(a_{k}\right) \exp \left(-a_{k}^{2} d\right)\right\} \\
F_{x 2}(x, d)=\left\{\sum_{k} A_{k} \cos \left(a_{k} x\right) f\left(a_{k}\right) g_{x 01}\left(a_{k}^{2} d\right)\right. \\
+\sum_{k} A_{k}^{2} \cos \left(a_{k} x\right) f\left(a_{k}\right) T_{x k}^{c}(d) \\
+\sum_{k<l} \sum_{k} A_{l}\left[\cos \left(a_{k} x\right) f\left(a_{l}\right)\right. \\
\left.\left.+\cos \left(a_{l} x\right) f\left(a_{k}\right)\right] T_{x k l}^{c}(d)\right\}
\end{gathered}
$$

$$
/\left\{\sum_{k} A_{k} \cos \left(a_{k} x\right) f\left(a_{k}\right) \exp \left(-a_{k}^{2} d\right)\right\}
$$

\section{DISCUSSIONS}

\section{Some Limiting Cases}

The value of $d$ increases as proportional to $D^{-2}$, with decreasing $D$. When $D$ is much smaller than the mean end-to-end distance in an unconfined state, only the contribution of the terms of $k=0$ is relevant in eq $28 \mathrm{a}$ and $28 \mathrm{~b}$. Using eq $25 \mathrm{a}$ and $25 \mathrm{~b}$, we get $p_{0}=x_{0} / d, p_{1}=$ $1 / 2$, and $a_{0}^{2} d p_{0}=a_{0}^{2} x_{0}$. All relevant terms in eq 28a are independent of $d$. Thus the terms in $F_{x 1}(d)$ are independent of $d$ except for $-\ln \left(p_{0}\right)$, which increases very gradually in proportion to $\log (d)$, with increasing $d$. In the same manner, we show that the value of $F_{x_{2}}(d)$ approaches the constant $+o(D)$, with decreasing $D$. Then eq 33 predicts that $\left\langle R_{x}^{2}\right\rangle$ increases in proportion to $D^{-1}$ with decreasing $D$ for any value of $W$, when the polymer is confined between two plates separated by a short distance. This prediction is consistent with the results obtained by Wang et al., ${ }^{6}$ and our previous calculations. ${ }^{7}$ However, this exponent differs from the prediction arrived at by scaling arguments ${ }^{4,5}$ or by the mean field approximation. ${ }^{8}$ One reason for this difference is that perturbation expansion cannot be applied when $D \ll\left\langle R_{z}^{2}\right\rangle_{\infty}^{1 / 2}$ because the values of the perturbation terms become very large.

When the limit to approach $D$ to infinity while $W$ is finite, the value of $d$ approaches 0 . The value of $F_{x 2}(d)$ becomes constant. Then the third term in [ ] of eq 33 is negligible. The value of $g_{x 00}\left(a_{k}^{2} d\right)$ becomes equal to $g_{\infty}$ for all values of $k$. The value of $\left\langle R_{x}^{2}\right\rangle$ approaches $\left\langle R_{x}^{2}\right\rangle_{\infty}$ as $D$ increases. However, when $W$ is negative, $a$ approaches $-D W$ as shown in Figure 5. $a^{2} d$ approaches $W^{2}\left\langle R_{z}^{2}\right\rangle / 2$, which is both positive and finite. The contribution of this term to $F_{x 1}(d)$ can be disregard in the calculation of $\left\langle R_{x}^{2}\right\rangle$, because the value of the coefficient, $A_{0} f^{2}\left(a_{0}\right) \exp \left(-a_{0}^{2} d\right)$ falls off to 0 
in proportion to $a^{-1}$ with increasing $a$. From eq $37 \mathrm{a}, F_{x 1}(z, d)$ can be rewritten as

$$
\begin{aligned}
& F_{x 1}(z, d)= \\
& \left\{A_{0} f_{0} \cosh (a z / D) \exp \left(a^{2} d\right) g_{x 00}\left(a^{2} d\right)\right. \\
& \left.\quad+\sum_{k} f\left(a_{k}\right) \cos \left(a_{k} z / D\right) \exp \left(a_{k}^{2} d\right) g_{x 00}(0)\right\} \\
& /\left\{A_{0} f_{0} \cosh (a z / D) \exp \left(a^{2} d\right)\right. \\
& \left.\quad+\sum_{k} f\left(a_{k}\right) \cos \left(a_{k} z / D\right) \exp \left(a_{k}^{2} d\right)\right\}
\end{aligned}
$$

where

$$
\begin{aligned}
& a=-D W \\
& A_{0}=\frac{1}{\sinh (a) / a+1}=2 a \exp (-a) \\
& f_{0}=\sinh (a / 2) /(a / 2)=\exp (a / 2) / a
\end{aligned}
$$

and

$$
a_{k}=(2 k-1) \pi \quad k>0
$$

for suitably large values of $a$. Using the relation

$\sum_{k}(-1)^{k} \frac{\cos (2 k-1) x}{2 k-1}= \begin{cases}\pi / 4 & x<\pi / 2 \\ 0 & x=\pi / 2\end{cases}$

we get

$$
\begin{aligned}
& F_{x 1}(z, d)= \\
& \quad\left\{\exp \left(w^{2} / 2-w r\right) g_{x 00}\left(-w^{2} / 2\right)+g_{\infty} / 4\right\} \\
& \quad /\left\{\exp \left(w^{2} / 2-w r\right)+1 / 4\right\}
\end{aligned}
$$

where

$$
w=-W\left\langle R_{z}^{2}\right\rangle_{\infty}^{1 / 2}
$$

and

$$
r=(D / 2-z) /\left\langle R_{z}^{2}\right\rangle_{\infty}^{1 / 2}
$$

Equation 41 shows that $F_{x 1}$ can be approximated as $g_{x 00}\left(-w^{2} / 2\right)$ when $r<w / 2$, and as $g_{\infty}$ when $r>w / 2$, respectively. The crossover region between the two states is narrow. Then the effective range of the polymer plate interaction is predicted to be $w / 2$ times as large as $\left\langle R_{z}^{2}\right\rangle_{\infty}^{1 / 2}$ when the interplate distance is large. Equation 41 also predicts that $\left\langle R_{x}^{2}(D / 2)\right\rangle$ is continuous at $W=0$, and no phase transition can be found from the viewpoint of chain dimensions.

\section{Crossover Behavior}

The values of $\left\langle R_{x}^{2}\right\rangle /\left\langle R_{x}^{2}\right\rangle_{\infty}$ are plotted against $D /\left\langle R_{z}^{2}\right\rangle_{\infty}^{1 / 2}$ for various values of $D W$ in Figure 7. Hereafter, the value of $\theta$ is placed at $1 / 2$. In the range of $D /\left\langle R_{z}^{2}\right\rangle_{\infty}^{1 / 2}<1$, the effect of confinement is important. The value of $\left\langle R_{x}^{2}\right\rangle /\left\langle R_{x}^{2}\right\rangle_{\infty}$ increases rapidly as $D /\left\langle R_{z}^{2}\right\rangle_{\infty}^{1 / 2}$ decreases for any value of $D W$. The segments of the confined chain are squeezed in a direction parallel to the plates, by compression of the chain in this region. The value of $\left\langle R_{x}^{2}\right\rangle$ decreases monotonously, and approaches the value for an unconfined state, as $D /\left\langle R_{z}^{2}\right\rangle_{\infty}^{1 / 2}$ increases. In the range of $\left.D /\left\langle R_{z}^{2}\right\rangle_{\infty}^{1 / 2}\right\rangle 1$, the effect of the repulsive polymer plate interaction becomes irrelevant. However the contribution of the attractive interaction remains relevant. $\left\langle R_{x}^{2}\right\rangle \mid\left\langle R_{x}^{2}\right\rangle_{\infty}$ is plotted against $D W$ in Figure 8 and increases with $D W$, when $D /\left\langle R_{z}^{2}\right\rangle_{\infty}^{1 / 2}$ is small and $W$, positive. This is explained as follows. The segments are pushed into the central part between the plates by the repulsive

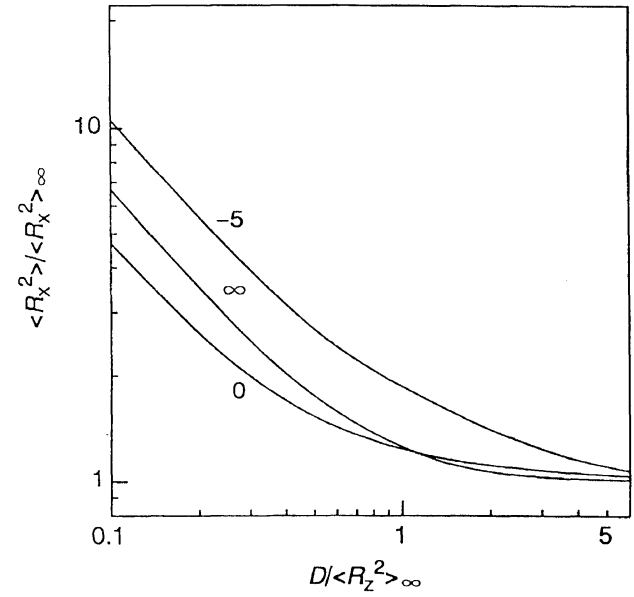

Figure 7. $\left\langle R_{x}^{2}\right\rangle /\left\langle R_{x}^{2}\right\rangle_{\infty}$ as functions of $D /\left\langle R_{z}^{2}\right\rangle_{\infty}^{1 / 2}$. The numerical values in the figure are the values of $D W$. 


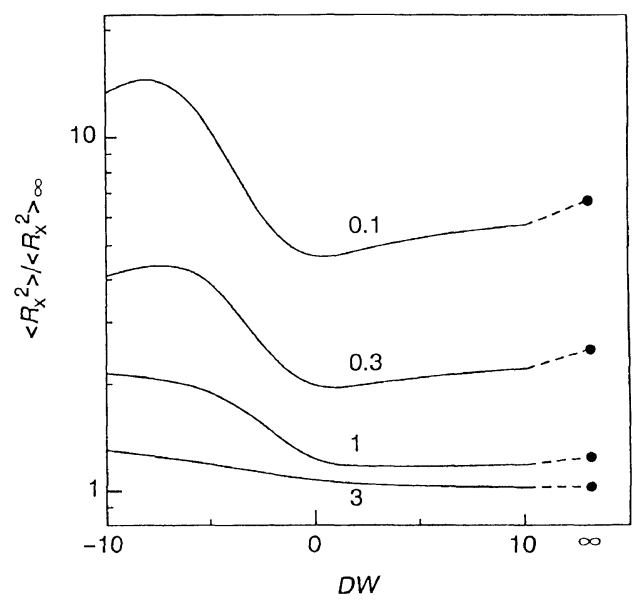

Figure 8. $\left\langle R_{x}^{2}\right\rangle /\left\langle R_{x}^{2}\right\rangle v s$. $D W$. The numerical values in the figure are the values of $D /\left\langle R_{z}^{2}\right\rangle_{\infty}^{1 / 2}$.

polymer plates interaction. While the interplate distance is large, $\left\langle R_{x}^{2}\right\rangle /\left\langle R_{x}^{2}\right\rangle_{\infty}$ is insensitive to $D W$. When $D W$ is negative, $\left\langle R_{x}^{2}\right\rangle \mid\left\langle R_{x}^{2}\right\rangle_{\infty}$ increases with decreasing $D W$. The interaction between the polymer and plates becomes more attractive, then the segments of the polymer are more adsorbed on the plate. The chain spreads along both plates when the chain dimension is much larger than $D$.

In Figures 9a and 9b, the values of $\left\langle R_{x}^{2}(z)\right\rangle /\left\langle R_{x}^{2}\right\rangle_{\infty}$ are plotted against $z / D$. The values of $\left\langle R_{x}^{2}(z)\right\rangle /\left\langle R_{x}^{2}\right\rangle_{\infty}$ are almost independent of $z$ for small values of $D /\left\langle R_{z}^{2}\right\rangle_{\infty}^{1 / 2}$. However, they vary with $z$ for large values of $D /\left\langle R_{z}^{2}\right\rangle_{\infty}^{1 / 2}$. It is interesting that the values of $\left\langle R_{x}^{2}(z)\right\rangle /\left\langle R_{x}^{2}\right\rangle_{\infty}$ of the chain where one end is anchored near the adsorbing plates are smaller than those of the chains where one end is located near the center between the plates. An intuitive interpretation is as follows. The chain segments are adsorbed by both plates when $D$ is small and $W$ is negative. When $D$ becomes larger than the chain dimensions, the bridge type conformation is elongated in a direction perpendicular to the plates; and the value of $\left\langle R_{x}^{2}(z)\right\rangle$ becomes smaller than those of other conformations. However, when $D$ is much larger than the chain dimensions, the chain is adsorbed by either of the two plates, and

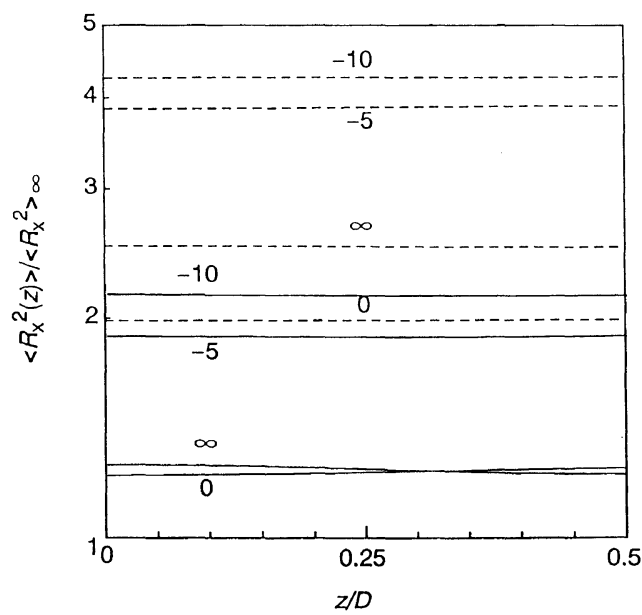

(a)

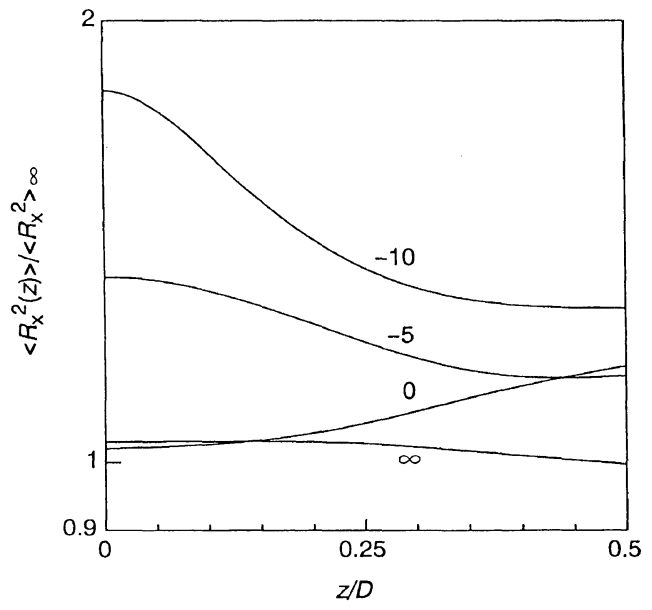

(b)

Figure 9. $\left\langle R_{x}^{2}(z)\right\rangle \mid\left\langle R_{x}^{2}\right\rangle_{\infty}$ as functions of $z$. Dotted curves, $D /\left\langle R_{z}^{2}\right\rangle_{\infty}^{1 / 2}=0.3$; solid curves in (a), $D /\left\langle R_{z}^{2}\right\rangle_{\infty}^{1 / 2}=1$; in (b); $D /\left\langle R_{z}^{2}\right\rangle_{\infty}^{1 / 2}=3$. The numerical values in the figure represent the values of $D W$.

$\left\langle R_{x}^{2}(D / 2)\right\rangle$ becomes larger than $\left\langle R_{x}^{2}(0)\right\rangle$.

Acknowledgment This work was supported in part by a Grant-in-Aid from the Ministry of Education, Science, and Culture of Japan.

\section{APPENDIX}

The explicit forms of $\mathrm{J}$ in eq 16 and 18 are as follows. 


$$
\begin{aligned}
J_{k m}^{c c}= & f\left(2 a_{k}\right) f\left(2 a_{m}\right)+\left[f\left(2 a_{k}-2 a_{m}\right)\right. \\
& \left.+f\left(2 a_{k}+2 a_{m}\right)\right] / 2 \\
J_{k m}^{c s}= & f\left(2 a_{k}\right) f\left(2 b_{m}\right)-\left[f\left(2 a_{k}-2 b_{m}\right)\right. \\
& \left.+f\left(2 a_{k}+2 b_{m}\right)\right] / 2 \\
J_{k m}^{s c}= & f\left(2 b_{k}\right) f\left(2 a_{m}\right)-\left[f\left(2 b_{k}-2 a_{m}\right)\right. \\
& \left.+f\left(2 b_{k}+2 a_{m}\right)\right] / 2 \\
J_{k m}^{s s}= & -f\left(2 b_{k}\right) f\left(2 b_{m}\right)+\left[f\left(2 b_{k}-2 b_{m}\right)\right. \\
& \left.+f\left(2 b_{k}+2 b_{m}\right)\right] / 2 \\
J_{k l m}^{c c}= & {\left[f\left(a_{k}-a_{l}-2 a_{m}\right)+f\left(a_{k}-a_{l}+2 a_{m}\right)\right.} \\
& \left.+f\left(a_{k}+a_{l}-2 a_{m}\right)+f\left(a_{k}+a_{l}+2 a_{m}\right)\right] / 2 \\
J_{k l m}^{c s}= & -\left[f\left(a_{k}-a_{l}-2 b_{m}\right)+f\left(a_{k}-a_{l}+2 b_{m}\right)\right. \\
& \left.+f\left(a_{k}+a_{l}-2 b_{m}\right)+f\left(a_{k}+a_{l}+2 b_{m}\right)\right] / 2 \\
J_{k l m}^{s c}= & {\left[f\left(b_{k}-b_{l}-2 a_{m}\right)+f\left(b_{k}-b_{l}+2 a_{m}\right)\right.} \\
& \left.-f\left(b_{k}+b_{1}-2 a_{m}\right)-f\left(b_{k}+b_{l}+2 a_{m}\right)\right] / 2
\end{aligned}
$$

$$
\begin{aligned}
J_{k l m}^{s s}= & -\left[f\left(b_{k}-b_{l}-2 b_{m}\right)+f\left(b_{k}-b_{l}+2 b_{m}\right)\right. \\
& \left.-f\left(b_{k}+b_{1}-2 b_{m}\right)-f\left(b_{k}+b_{1}+2 b_{m}\right)\right] / 2
\end{aligned}
$$

\section{REFERENCES}

1. For examples, A. Takahashi and M. Kawaguchi, $A d v$. Polym. Sci., 46, 1 (1982).

2. E. A. DiMarzio and R. J. Rubin, J. Chem. Phys., 55, 4318 (1971).

3. D. Chan, B. Davies, and P. Richmond, J. Chem. Soc., Faraday Trans. 2, 72, 1584 (1975).

4. M. Daoud and P. G. de Gennes, J. Physiques, 38, 85 (1977).

5. L. Turban, J. Physiques, 45, 341 (1984).

6. Z. Wang, A. M. Nemirovsky, and K. F. Freed, J. Chem. Phys., 86, 4266 (1987).

7. K. Shiokawa, Polym. J., 22, 925 (1990).

8. K. Shiokawa, Polym. J., 23, 885 (1991).

9. Y. Oono, Phys. Rev. A, 30, 986 (1984).

10. P. G. de Gennes, Rep. Prog. Phys., 32, 187 (1969). 